\section{Les édulcorants de la controverse}

Alexandra Pepin, Pascal Imbeault

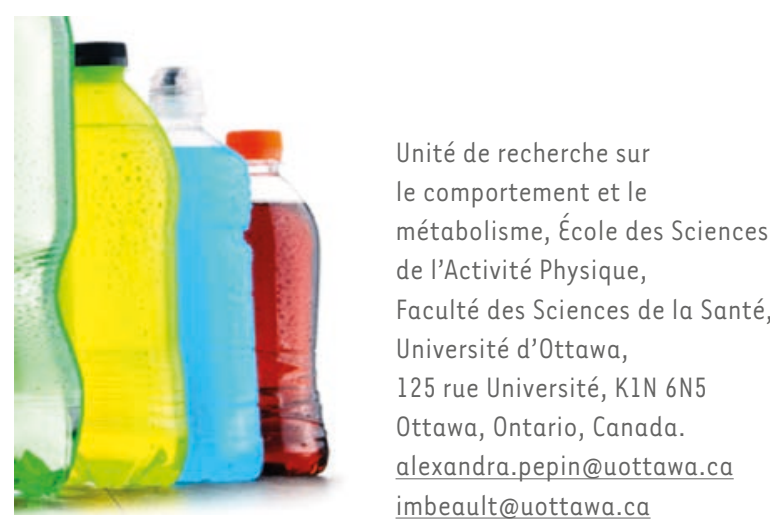

alimentaires englobent à la fois les édulcorants artificiels et les édulcorants d'origine naturelle, tels que les extraits purifiés de stévia ${ }^{1}$ et de fruit du moine ${ }^{2}$ [5]. Substituts des sucres ajoutés, ils procurent la sensation de goût sucré tout en ne contenant peu ou pas de calories et sont communément utilisés comme additifs dans les produits alimentaires, tels que les boissons, les bonbons, les yogourts, les fruits en conserve et les céréales à déjeuner [5]. Ils peuvent également être obtenus en vente libre par les consommateurs [5]. Dans cette revue, nous examinerons si l'utilisation des édulcorants présente un risque pour la santé métabolique. Plus précisément, nous discuterons des effets potentiels de la consommation de substituts de sucre sur l'apport énergétique, sur le microbiote intestinal et sur le métabolisme du glucose. Finalement, nous analyserons les édulcorants comme outil nutritionnel et s'ils méritent d'être recommandés par les professionnels de la santé à leurs patients.

\section{L'innocuité des substituts de sucre : qui la détermine et comment?}

À l'échelle mondiale, une panoplie de substituts de sucres sont approuvés pour l'utilisation alimentaire, dont les suivants: l'acésulfame-potassium (Ace-K), l'advantame, l'aspartame, le néotame, la saccharine, le sucralose, la thaumatine, les glycosides de stéviol, les extraits de fruit du moine, ainsi que les sucres alcool (polyalcools ou polyols), tels que le lactitol, l'isomalt, le mannitol, le sorbitol et le xylitol [6]. Leur avantage principal repose sur leur pouvoir sucrant

\footnotetext{
${ }^{1}$ II s'agit d'extrait naturel de plante utilisé comme édulcorant naturel par les communautés indigènes d'Amérique du Sud depuis plusieurs siècles. Les glycosides de stéviol sont réglementés comme additifs alimentaires. Cependant, les feuilles de Stévia sont considérées comme un ingrédient alimentaire, et non comme un additif alimentaire. Peu de réglementations existent donc sur l'utilisation des feuilles de stévia dans les aliments et les boissons emballés.

${ }^{2}$ Ce petit fruit ovale (Luo Han Guo) poussant au sud de la Chine et dans le nord de la Thaillande est utilisé comme édulcorant et ingrédient fonctionnel dans la médecine traditionnelle chinoise. Les moines bouddhistes le cultivaient pour ses vertus médicinales, d'où son nom de fruit du moine.
} 
très élevé comparé au sucrose, soit le traditionnel sucre blanc [7]. De minimes quantités d'édulcorants sont donc suffisantes pour obtenir le même goût sucré. Par exemple, le pouvoir sucrant de l'aspartame et du sucralose est perçu respectivement 200 fois et 600 fois plus sucré que celui du sucrose pour une même quantité. Bien que les sucres alcool soient également des substituts de sucre, ils ne sont pas considérés comme des édulcorants de haute intensité, étant donné que leur pouvoir sucrant est similaire ou inférieur à celui du sucrose [8]. Le Tableau / présente différents substituts de sucre et permet de comparer leurs profils en termes de pouvoir sucrant, d'apport quotidien acceptable et la façon dont ils sont métabolisés par notre organisme. Les substituts de sucre sont soumis à des réglementations très strictes dans le but d'assurer qu'ils ne sont pas nocifs pour la santé humaine [5]. Les étapes qui précèdent l'approbation d'un substitut de sucre par un organisme de réglementation fédéral ou gouvernemental sont mal comprises du public et méritent d'être mises en lumière. Bien avant son approbation, des études précliniques rigoureuses sont entreprises afin de déterminer la toxicocinétique (examen de l'absorption, de la distribution, du métabolisme et de l'excrétion d'une substance donnée) de l'édulcorant et d'en assurer son innocuité [9]. Ces études précliniques d'innocuité sont réalisées chez l'animal et utilisent un large éventail de concentrations afin de parvenir à évaluer la relation dose-effet qui pourrait engendrer des effets néfastes pour la santé. La dose la plus élevée qui ne provoque pas d'effets biologiques, lorsqu'elle est consommée dans le cadre d'études à doses répétées de longue durée, est appelée Dose Sans Effet Indésirable Observé [9]. Afin de pouvoir extrapoler les résultats de ces études conduites chez l'animal, à l'humain, la valeur de la Dose Sans Effet Indésirable Observé est ensuite divisée par un facteur de sécurité (pour les substituts de sucre, ce facteur est souvent de 100) afin de garantir une marge de sécurité conservatrice [9]. Ce calcul est utilisé pour établir un apport quotidien admissible (APA) chez l'humain, étant défini comme «la quantité d'additif alimentaire [dans ce cas d'un substitut de sucre $\mathrm{X}$ ], exprimée en $\mathrm{mg} / \mathrm{kg}$ de poids corporel/jour, pouvant être ingérée quotidiennement dans une vie sans aucun risque appréciable pour la santé » [10]. Il est très peu probable de dépasser ou même d'atteindre I'AQA de la plupart des édulcorants, principalement en raison des quantités minimes d'édulcorants requises pour obtenir un goût sucré appréciable, mais aussi parce que les industries utilisent souvent une combinaison de plusieurs édulcorants dans le but de réduire la quantité de chacun dans la formulation de leurs produits. Ainsi, pour atteindre l'APA de l'aspartame, soit $50 \mathrm{mg} / \mathrm{kg}$ de poids corporel/jour, il serait nécessaire de consommer environ 18 cannettes de boissons gazeuses «diète » contenant de l'aspartame comme seul édulcorant, et ce en une journée [11]. Cependant, cette quantité, qui peut paraître élevée, ne l'est pas forcément pour tous les édulcorants. En effet, l'AQA du sucralose est de $5 \mathrm{mg} / \mathrm{kg}$ de poids corporel/jour [7]. Sachant qu'une cannette de $355 \mathrm{~mL}$ de boisson gazeuse sans caféine «diète » d'une marque populaire contient $68 \mathrm{mg}$ de sucralose et $41 \mathrm{mg}$ d'acésulfame-potassium [12], il suffirait donc de 5 cannettes pour qu'un individu ayant un poids corporel de $70 \mathrm{~kg}$ atteigne I'AQA du sucralose. Bien que des réglementations strictes et des études rigoureuses assurent l'innocuité des substituts de sucre au préalable à leur approbation pour utilisation comme additif alimentaire, les études toxicocinétiques ne permettent cependant pas de tirer des conclusions quant aux conséquences métaboliques qui pourraient être associées à la consommation de ces substituts. En d'autres termes, le simple fait que les substituts de sucre sont en effet considérés comme étant «sans danger » pour la santé humaine (absence de toxicité ou de risque de causer des maladies, telles que le cancer) ne signifie pas qu'ils constituent une option «saine » pour le consommateur (état physiologique optimal pour maintenir une bonne santé) [13]. De ce fait, un grand nombre de professionnels de la santé hésitent donc à recommander la prise d'édulcorants à titre d'outil nutritionnel favorisant la gestion du poids corporel, et ce principalement en raison des incertitudes quant aux effets de leur consommation à long terme sur la santé métabolique [14].

\section{Les substituts de sucre et la gestion du poids corporel : ce qu'en dit la science...}

Ce n'est pas d'hier que des préoccupations en lien avec les effets de la consommation d'édulcorants sur le métabolisme et la régulation des mécanismes de l'appétit ont été exprimées. Des études observationnelles ont en effet révélé que l'augmentation substantielle de la consommation d'édulcorants au cours des quatre dernières décennies coïncide avec l'accroissement de la prévalence et de l'incidence de l'obésité [15-17], ouvrant ainsi la porte à suspecter que la prise de substituts de sucre pourrait altérer certains mécanismes régulant l'apport énergétique. Néanmoins, des essais randomisés contrôlés, qui ont un pouvoir statistique plus fort que les études observationnelles, ne supportent pas cette idée selon laquelle l'apport en substituts de sucre favoriserait une augmentation de l'apport énergétique. Une méta-analyse récente, incluant des études conduites chez des populations d'adultes et d'enfants pendant une durée de 3 à 78 semaines, révèle que la substitution du sucre par des édulcorants entraîne une perte de poids modeste inférieure à 1 kg [18]. À la lumière de ces résultats, il fut conclu que l'utilisation d'édulcorants pouvait donc être recommandée chez les individus visant une perte ou un maintien de perte de poids corporel [18]. Cependant, comme les études comprises dans cette méta-analyse sont toutes des interventions ayant comparé la consommation d'édulcorants à celle de sucre, on ne peut déterminer si les effets observés au niveau du poids corporel sont directement reliés à la 


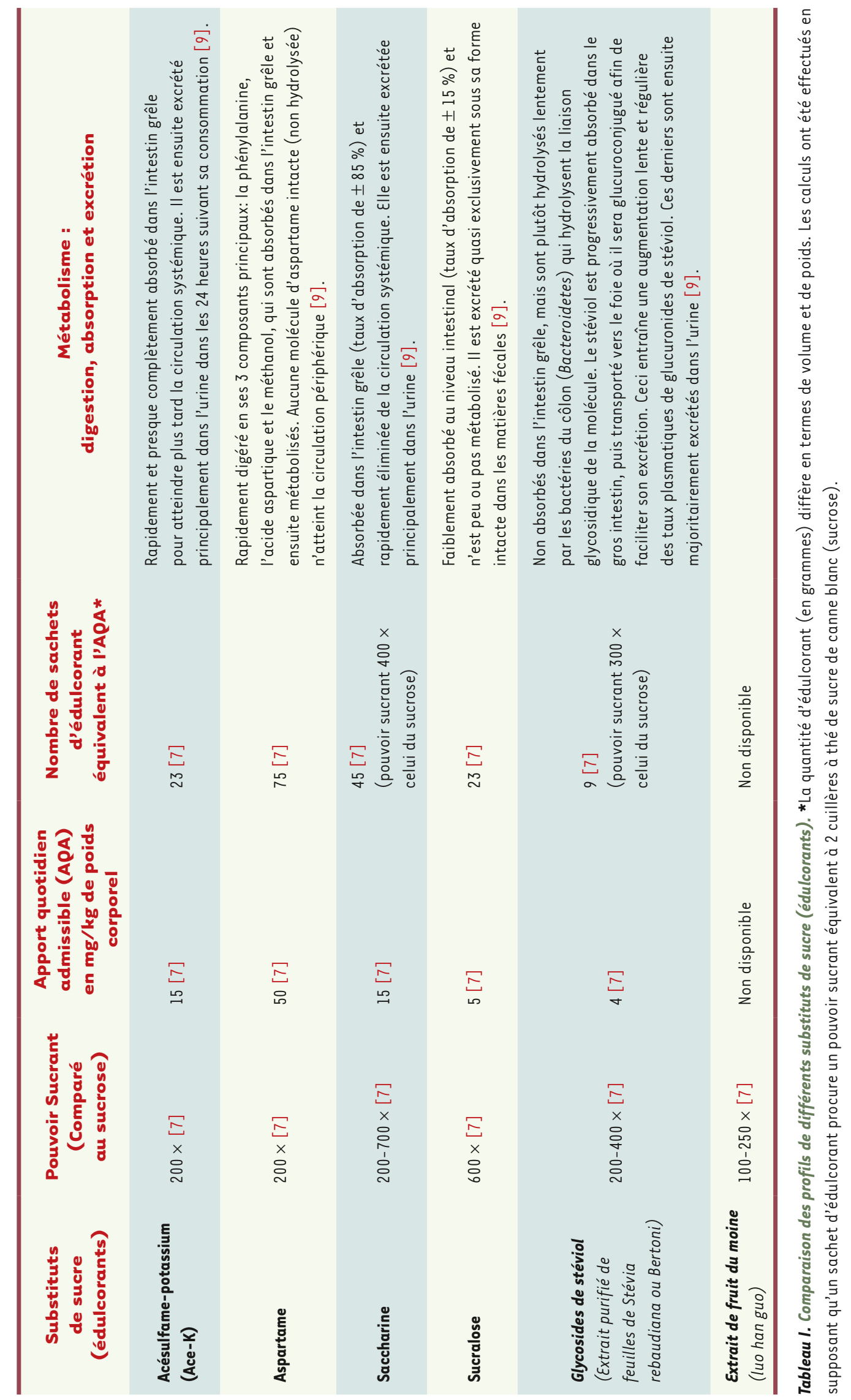


consommation d'édulcorants. Autrement dit, il est possible qu'une perte de poids similaire soit observée en réduisant la consommation de sucre sans nécessairement avoir à substituer le sucre dans l'alimentation par des édulcorants. II faut également noter que cette méta-analyse a reçu un soutien financier de la branche nord-américaine de I'International Life Sciences Institute (Institut International des Sciences Humaines), une organisation internationale à but non lucratif, mais financée et fortement influencée par les industries alimentaires [19-21]. Une revue systématique d'auteurs ayant bénéficié de subventions de recherche par l'industrie du sucre n'a également pas relevé d'évidence chez l'humain supportant l'augmentation de l'apport énergétique et du poids corporel en lien avec la consommation de substituts de sucre [22]. Fait intéressant, les auteurs de cette étude ont également stipulé que l'utilisation de boissons sucrées à base d'édulcorants semblait neutre ou même bénéfique à la gestion du poids corporel, et ce même en comparaison à de l'eau [22]. Toutefois, cette revue systématique a regroupé les interventions d'une durée de plus de 24 heures comme étant des «interventions prolongées », cette durée étant nettement insuffisante pour observer les effets chroniques de l'utilisation de substituts de sucre au niveau de la gestion du poids corporel. Ainsi, les conclusions issues des résultats de cette revue systématique ne reposent pas sur des données représentatives d'une consommation à long terme de substituts de sucre et ne peuvent donc pas être interprétées comme telles.

\section{Les effets paradoxaux des substituts de sucre}

Bien que les résultats issus d'études observationnelles ne permettent pas d'établir une relation de cause à effet entre la consommation de substituts de sucre et le gain de poids corporel, certains mécanismes par lesquels les édulcorants pourraient favoriser un gain pondéral à long terme ont été proposés. II est d'ailleurs suggéré que la prise de substituts de sucre pourrait maintenir et renforcer le désir pour les aliments sucrés, pouvant ainsi compromettre la perte ou le maintien du poids corporel à long terme. La consommation d'édulcorants pourrait également mener à la production de signaux d'appétit contradictoires dû au fait qu'ils sont perçus comme une source de calories par nos papilles gustatives, alors qu'ils en sont exempts. Ces «faux messages » pourraient en fait résulter en une augmentation ultérieure de l'appétit et subséquemment l'augmentation de l'apport énergétique, phénomène présenté comme un des effets paradoxaux des substituts de sucre [23].

Les stimulations orosensorielles qui sont induites lorsque nous consommons des aliments ou des boissons qui procurent à la fois un goût sucré et un contenu calorique initient des mécanismes impliqués dans la régulation de l'apport énergétique. Parmi ceux-ci, l'alliesthésie alimentaire négative est une sensation qui prévient la surconsommation des aliments sucrés et/ou gras en diminuant le plaisir perçu lorsqu'on les consomme, ou après les avoir ingérés [23]. Ainsi, l'alliesthésie alimentaire négative est susceptible d'être affaiblie par la consommation d'édulcorants si l'on prend en considération le fait que le goût sucré de ces derniers n'est pas associé à un apport énergétique [23-25]. De plus, l'ingestion répétée de substituts de sucre, qui fournit le stimulus orosensoriel du goût sucré sans toutefois être une source d'énergie considérable, pourrait également perturber l'équilibre énergétique en diminuant la capacité du goût sucré à prédire le contenu calorique d'un aliment ou d'une boisson, conduisant ainsi à une diminution des réponses de la phase céphalique [26]. Les réponses de la phase céphalique sont une variété de réponses hormonales, neuronales et métaboliques induites par les stimulus orosensoriels (par exemple, la salivation, la sécrétion d'acide gastrique, la thermogenèse et la sécrétion d'hormones telles que l'insuline, la ghréline et la leptine) qui facilitent le transit des aliments ingérés dans le tractus gastro-intestinal $[27,28]$ et qui sont également impliquées dans la régulation de l'appétit et de la satiété [29]. Selon cette approche, l'altération des réponses de la phase céphalique par les édulcorants aurait ainsi pour effet de stimuler l'apport énergétique spécifiquement pour les aliments sucrés et caloriques, ceci pouvant contribuer à un gain de poids non désiré [26]. Quoique prometteur, il est important de souligner que ces hypothèses ont été confirmées par des études réalisées chez le rongeur [30]. II reste maintenant à savoir si ces résultats peuvent être transposés à l'humain.

\section{Les substituts de sucre : ennemis du microbiote?}

En plus de fournir très peu de calories, les substituts de sucre sont également intéressants pour les patients diabétiques puisqu'ils n'ont aucun effet sur la glycémie... du moins c'est ce que nous pensions. La plupart des édulcorants ne sont pas digérés dans l'intestin grêle (les détails quant au métabolisme de digestion, d'absorption et d'excrétion de divers édulcorants sont présentés dans le Tableau /) et peuvent par conséquent rencontrer le microbiote intestinal. La composition du microbiote intestinal est une signature unique qui peut pratiquement être comparée à une empreinte digitale. $\varepsilon$ n tant qu'écosystème complexe et dynamique, le microbiote diffère grandement entre les individus et est directement influencé par certains facteurs propres à l'hôte qui l'héberge, comme la génétique [31] et l'âge [32], mais aussi par des facteurs environnementaux, tels que l'exposition aux antibiotiques [33], les habitudes de vie [33] et de manière encore plus importante, par l'alimentation $[34,35]$. Des preuves convaincantes démontrent que les altérations du microbiote intestinal ont des effets néfastes sur la physiologie de l'hôte et contribuent, comme facteur environnemental, au développement de l'obésité, de l'inflammation de faible grade et de la résistance à l'insuline $[13,36-38](\rightarrow)$.

$(\rightarrow)$ Voir la Nouvelle de B. Chassaing, $m / s$ $n^{\circ}$ 6-7, juin-juillet 2015, page 586 
Selon ses interactions avec l'environnement, ce microbiote est sujet à une dysbiose, soit une perturbation de l'homéostasie intestinale causée par un déséquilibre dans la configuration structurelle et/ou fonctionnelle des microorganismes bénéfiques et protecteurs qui le composent [39]. Comme cette voie de recherche est très récente, les mécanismes qui sous-tendent l'implication du microbiote dans la modulation des réponses glycémiques chez l'humain n'ont pas encore été clairement établis [37]. Toutefois, plusieurs mécanismes ont été proposés et sont au cœur des recherches actuelles dont la production de peptides intestinaux qui stimuleraient la sécrétion d'insuline par les cellules bêta du pancréas et la production par les bactéries intestinales d'acide gras à chaîne courte présentant des effets bénéfiques au niveau de l'inflammation et de l'intégrité de la barrière muqueuse intestinale [37].

La relation entre le microbiote intestinal et le métabolisme du glucose a notamment été examinée par le transfert du microbiote intestinal, c'est-à-dire la greffe de matières fécales, de donneurs sains à des sujets atteints du syndrome métabolique. Celle-ci a montré une amélioration significative de la sensibilité à l'insuline des patients transplantés, après six semaines d'intervention [36].

Les travaux de Suez et al. en 2014 [40] réalisés chez le rongeur ont permis de démontrer que la consommation de saccharine, de sucralose, ou d'aspartame, à des doses équivalentes à l'AQA durant 11 semaines, produisait une dysbiose et une diminution significative de la tolérance au glucose, celle-ci étant associée avec un état prédiabétique [41]. Parallèlement, la greffe de matières fécales provenant de souris ayant consommé une solution commerciale de saccharine transférée à des souris microbiologiquement stériles a également résulté en une altération de la tolérance au glucose, suggérant ainsi un rôle important du microbiote dans l'intolérance au glucose [40]. En poursuivant leurs observations chez l'humain, la consommation de saccharine à des doses équivalentes à l'AQA a résulté en une dysbiose, en plus d'une intolérance au glucose chez la moitié des sujets étudiés en sept jours seulement, alors que l'autre moitié des sujets n'ont pas présenté d'altération [40]. Les auteurs ont d'ailleurs souligné qu'il semble exister des différences interindividuelles importantes dans les réponses glycémiques et le profil du microbiote suivant la consommation de substituts de sucre, suggérant ainsi que la consommation d'édulcorants n'affecterait pas tous les individus de la même façon et que les réponses métaboliques pourraient être influencées par la composition initiale de leur microbiote [40]. Afin d'identifier les réponses métaboliques pouvant être propres à chaque variété de substituts de sucre, une intervention visant à étudier les effets sur 7 jours de doses de sucralose équivalentes à approximativement $75 \%$ de l'APA sur le microbiote intestinal et le métabolisme du glucose a été réalisée chez l'humain. Aucune différence significative n'a été détectée entre les résultats du groupe expérimental et ceux du groupe contrôle en ce qui concerne la diversité du microbiote intestinal et de la résistance à l'insuline [42]. Dans l'ensemble, ces résultats suggèrent que les effets métaboliques associés à la consommation de substituts de sucre peuvent varier en fonction de l'individu, de la variété d'édulcorant utilisée et du temps d'exposition. Les deux études précédentes font partie des très rares interventions qui ont examiné les effets métaboliques de la consommation d'édulcorants pendant plus d'une semaine. Une méta-analyse récente a par ailleurs conclu que la consommation d'édulcorants (aspartame, saccharine, sucralose et stévia) n'avait pas d'effet significatif sur les réponses glycémiques chez l'humain [43]. Néanmoins, seuls 3 des 29 essais randomisés contrôlés inclus dans cette méta-analyse ont exposé les participants aux édulcorants durant plus d'une journée (deux interventions avec une durée d'exposition de 3 jours $[44,45]$ et une avec une durée d'exposition de 2 semaines [46]). Les résultats issus de cette méta-analyse doivent donc être interprétés avec prudence puisqu'ils ne sont pas représentatifs d'une consommation à long terme de substituts de sucre.

\section{Ce qu'il faut en retenir...}

Ne compromettant pas le goût sucré des aliments et des boissons, les substituts de sucre semblent être une alternative prometteuse pour une utilisation par des individus qui visent une restriction calorique ou qui désirent maintenir une perte de poids à long terme. Cette affirmation suscite la controverse dans la sphère scientifique puisque la consommation de substituts de sucre a été impliquée dans la perturbation de mécanismes de régulation de l'appétit, pouvant ainsi conduire à un apport calorique accru et à une prise de poids ultérieure. La plupart des mécanismes par lesquels cela pourrait se produire ne sont cependant pas supportés par des preuves convaincantes à ce jour. Bien qu'il soit incertain que les substituts de sucre favorisent l'apport alimentaire, on ne peut non plus conclure qu'ils favorisent la perte de poids. Des études récentes suggèrent que la consommation de certaines variétés d'édulcorants altère la diversité et la richesse du microbiote intestinal, ayant pour effet d'entraîner une perturbation des réponses glycémiques. Une question persiste: quelle option est la meilleure pour la santé, le sucre ou les substituts de sucre? La réponse est : aucune. L'idée de considérer les substituts de sucre comme une alternative saine aux sucres libres et ajoutés, sous prétexte qu'ils ne contiennent peu ou pas de calories, constitue une solution trop simple à un problème beaucoup plus complexe. La surconsommation de sucres libres et ajoutés est clairement associée à des effets néfastes pour la santé. Cependant, il n'y a pas suffisamment de preuves supportant que l'utilisation d'édulcorants soit sans conséquence sur la santé métabolique et physiologique. Sur la base des preuves disponibles, il est donc difficile d'endosser la recommandation des substituts de sucre comme outil 
nutritionnel dans le contexte de la gestion du poids corporel. L'utilisation d'édulcorants n'est pas nécessaire pour réduire l'apport en sucre ni pour réduire l'apport énergétique. II serait plus bénéfique de mettre l'accent sur une réduction graduelle de la consommation quotidienne de sucres libres et ajoutés, tout en faisant valoir les avantages pour la santé de consommer des aliments et des boissons non transformés. Il existe un dicton célèbre voulant qu'il suffise de 21 jours pour briser une habitude [47] (en fait, scientifiquement parlant, il faudrait en moyenne 66 jours pour en former une nouvelle [48]). Nous croyons que cela vaut la peine d'essayer. $\diamond$

\section{SUMMARY}

The controversial effects of low-calorie sweeteners

Substantial reviews of the most recent evidence report a strong relationship between the consumption of sugar-sweetened beverages and adverse health effects, such as the prevalence of obesity and metabolic diseases. Various public policies were recently undertaken by many countries in the hope of reducing sugar-sweetened beverage consumption amongst their population. This has led the food industries to promote low-calorie sweeteners (sugar substitutes) as a healthy alternative that would limit caloric intake without compromising the sweet taste of food and beverages. However, is the use of low-calorie sweeteners as a means of limiting or reducing energy intake without consequences for our health? This review aims to discuss the effects of low-calorie sweeteners consumption on health and to elucidate whether their use should be recommended by health professionals to their patients as part of weight management. $\diamond$

\section{LIENS D'INTÉRÊT}

Les auteurs déclarent n'avoir aucun lien d'intérêt concernant les données publiées dans cet article.

\section{RÉFÉRENCES}

1. Malik VS, Schulze MB, Hu FB. Intake of sugar-sweetened beverages and weight gain: a systematic review 1-3. Am J Clin Nutr $2006 ; 84: 274-288$.

2. Tappy L, Lê KA. Metabolic effects of fructose and the worldwide increase in obesity. Am Physiol Soc $2010 ; 90: 23-46$.

3. Pepin A, Stanhope KL, Imbeault P. Are fruit juices healthier than sugar-sweetened beverages? A review. Nutrients $2019 ; 11: 1006$.

4. World Health Organization. Guideline sugars intake for adults and children. Geneva : WHO, $2015: 50$.

5. Santé Canada. Succédanés du sucre. 2010 ; 1-5.

6. Santé Canada. Liste des édulcorants autorisés (listes des additifs alimentaires autorisés). 2019 ; $1-20$.

7. US Food and Drug Administration. Additional information about high-intensity sweeteners permitted for use in food in the United States. 2018 ; 1-5.

8. Santé Canada. Polyalcools (polyols) et polydextrose utilisés dans les aliments. 2005 ; 1-5.

9. Magnuson BA, Carakostas MC, Moore NH, et al. Biological fate of low-calorie sweeteners. Nutr Rev $2016 ; 74: 670-89$.

10. World Health Organization. Principles for the safety assessment of food additives and contaminants in food. Geneva : WHO, $1987: 124 \mathrm{p}$.

11. Mattes RD, Popkin BM. Nonnutritive sweetener consumption in humans: effects on appetite and food intake and their putative mechanisms. Am J Clin Nutr $2009 ; 89: 1-14$.

12. Sylvetsky AC, Brown RJ, Blau JE, et al. Hormonal responses to non-nutritive sweeteners in water and diet soda. Nutr Metab $2016 ; 13: 1-8$.

13. Nettleton JE, Reimer RA, Shearer J. Reshaping the gut microbiota: impact of low calorie sweeteners and the link to insulin resistance? Physiol Behav $2016 ; 164: 488-93$.

14. Harricharan M, Wills J, Metzger N, et al. Dietitian perceptions of low-calorie sweeteners. Eur J Public Health $2015 ; 25: 472-6$.

15. Stellman SD, Garfinkel L. Artificial sweetener use and one-year weight change among women. Prev Med (Baltim) 1986; $15: 195-202$.
16. Fowler SP, Williams K, Resendez RG, et al. Fueling the obesity epidemic? Artificially sweetened beverage use and long-term weight gain. Obesity $2008 ; 16: 1894-900$.

17. Sylvetsky AC, Jin Y, Mathieu K, et al. Low-calorie sweeteners: disturbing the energy balance equation in adolescents? Obesity $2017 ; 25: 2049-54$.

18. Miller PE, Perez V. Low-calorie sweeteners and body weight and composition: a meta-analysis of randomized controlled trials and prospective. Am J Clin Nutr $2014 ; 100: 765-77$.

19. Mckee M, Steele S, Stuckler D. The hidden power of corporations. BMJ 2019 ; $364: 9-10$.

20. Greenhalgh $\mathrm{S}$. Soda industry influence on obesity science and policy in China.J Public Health Policy 2019; $40: 5-16$.

21. US Right to Know. International Life Sciences Institute (ILSI) is a food industry lobby group. 2019 ; 1-7.

22. Rogers PJ, Hogenkamp PS, Graaf C de, et al. Does low-energy sweetener consumption affect energy intake and body weight? A systematic review, including meta-analyses, of the evidence from human and animal studies. Int J Obes $2016 ; 40: 381-94$.

23. Blundell JE, Hill AJ. Paradoxical effects of an intense sweetener (aspartame) on appetite. Lancet 1986 ; 1092-3.

24. Blundell JE, Rogers PJ, Hill AJ. Uncoupling sweetness and calories: methodological aspects of laboratory studies on appetite control. Appetite 1988 ; $11: 54-61$.

25. Rogers PJ, Blundell JE. Separating the actions of sweetness and calories: effects of saccharin and carbohydrates on hunger and food intake in human subjects. Physiol Behav $1989 ; 45$ : $1093-9$.

26. Davidson TL, Swithers SE. A Pavlovian approach to the problem of obesity. Int J Obes $2004 ; 28: 933-5$.

27. Power ML, Schulkin J. Anticipatory physiological regulation in feeding biology: cephalic phase responses. Appetite 2008 ; 50 : 194-206.

28. Woods SC, Ramsay DS. Pavlovian influences over food and drug intake. Behav Brain Res $2000 ; 110$ : 175-82.

29. Smeets PAM, Erkner A, Graaf C De. Cephalic phase responses and appetite. Nutr Rev $2010 ; 68: 643-55$.

30. Swithers SE, Martin AA, Davidson TL. High-intensity sweeteners and energy balance. Physiol Behav $2010 ; 100: 55-62$.

31. Zoetendal $\varepsilon G$, Akkermans ADL, Akkermans-van Vliet WM, et al. The host genotype affects the bacterial community in the human gastrointestinal tract. Microb Ecol Health Dis 2001 ; 13 : 129-34.

32. Hopkins MJ, Sharp R, Macfarlane GT. Age and disease related changes in intestinal bacterial populations assessed by cell culture, 16S rRNA abundance, and community cellular fatty acid profiles. Gut $2001 ; 48: 198-205$.

33. O'Hara AM, Shanahan F. The gut flora as a forgotten organ. EMBO Rep 2006 ; $7: 688-93$.

34. Graf D, Cagno R Di, Fåk F, et al. Contribution of diet to the composition of the human gut microbiota. Microb Ecol Heal Dis $2015 ; 26: 1-11$.

35. Davenport ER, Mizrahi-Man 0, Michelini K, et al. Seasonal variation in human gut microbiome composition. PLoS One 2014 ; 9.

36. Vrieze A, Nood $\varepsilon$ Van, Holleman F, et al. Transfer of intestinal microbiota from lean donors increases insulin sensitivity in individuals with metabolic syndrome. Gastroenterology 2012 ; 143 : 913-6.e7.

37. Gérard C, Vidal H. Impact of gut microbiota on host glycemic control. Front Endocrinol (Lausanne). $2019 ; 10: 1-13$.

38. Chassaing B. Rôle de certains additifs alimentaires dans l'apparition d'une inflammation intestinale et du syndrome métabolique chez la souris. Med Sci (Paris) $2015 ; 31: 586-8$.

39. Sommer F, Bäckhed F. The gut microbiota-masters of host development and physiology. Nat Rev Microbiol 2013 ; 11 : 227-38.

40. Suez J, Korem T, Zeevi D, et al. Artificial sweeteners induce glucose intolerance by altering the gut microbiota. Nature $2014 ; 514: 181-6$.

41. O'Rahilly S, Gray H, Hattersley A, et al. Insulin resistance as the major cause of impaired glucose tolerance: a self-fulfilling prophesy? Lancet $1994 ; 344: 585-9$.

42. Thomson P, Santibañez R, Aguirre C, et al. Short-term impact of sucralose consumption on the metabolic response and gut microbiome of healthy adults. BrJ Nutr $2019 ; 1-23$.

43. Nichol AD, Holle MJ, An R. Glycemic impact of non-nutritive sweeteners: a systematic review and meta-analysis of randomized controlled trials. Eur J Clin Nutr $2018 ; 72: 796-804$.

44. Shigeta $H$, Yoshida T, Nakai M, et al. Effects of aspartame on diabetic rats and diabetic patients. J Nutr Sci Vitaminol (Tokyo) $1985 ; 31: 533-40$.

45. Geuns JMC, Buyse J, Vankeirsbilck A, et al. Metabolism of stevioside by healthy subjects. Exp Biol Med 2007 ; 232 : 164-73. 


\section{RÉFÉRENCES}

46. Okuno G, Kawakami F, Tako H, et al. Glucose tolerance, blood lipid, insulin and glucagon concentration after single or continuous administration of aspartame in diabetics. Diabetes Res Clin Pract $1986 ; 2: 23-7$.

47. Maltz M. Psycho-cybernetics. New York : Essandess Special Editions, 1960.

48. Lally P, Jaarsveld CHM Van, Potts HWW, et al. How habits are formed: modelling habit formation in the real world. Eur J Soc Psychol $2010 ; 40$ : 998-1009.

49. Mooradian AD, Smith M, Tokuda M. The role of artificial and natural sweeteners in reducing the consumption of table sugar: A narrative review. Clin Nutr ESPEN $2017 ; 18: 1-8$.

TIRÉS À PART

A. Pepin

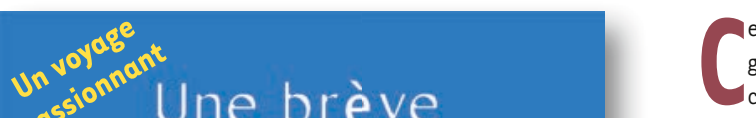

intéressant et lisible à la fois pour le spécialiste et le rand public, apporte des observations originales et nouvelles concernant l'angiogenèse, et notamment l'histoire des différentes découvertes, et discute les aspects et les concepts plus généraux en les plaçant dans le contexte de la philosophie des sciences.

Facile à lire, bien illustré, cet ouvrage cherche à comprendre et à faire comprendre les enjeux de la recherche sur l'arbre vasculaire en développement et en pathologie. II intéressera non seulement les étudiants et post-doctorants en biologie, mais aussi les chercheurs actifs dans ce domaine de recherche ainsi que toute personne intéressée par la biologie et la médecine et par l'histoire des sciences. Un voyage passionnant à travers l'histoire et les concepts les plus actuels concernant les recherches sur le vaisseau sanguin.

Andreas Bikfalvi est Professeur à l'université de Bordeaux et Directeur d'une unité de recherche Inserm sur le cancer et la biologie vasculaire. Il est, par ailleurs, membre senior de l'Institut Universitaire de France (IUF) et reconnu internationalement pour ses recherches dans le domaine de l'angiogenèse tumorale.
ISBN : 978-2-7598-1863-1$$
8<
$$

À retourner à EDP Sciences, 17, avenue du Hoggar, 91944 Les Ulis Cedex, France

Tél. : 0149856069 - Fax : 0149850345 - $\varepsilon$-mail : françois.flori@edpsciences.org

NOM :

Prénom

Adresse :

Code postal :

Ville :

Pays :

Fonction :

Je souhaite recevoir

Une brève histoire de vaisseau : $25 €+3 €$ de port $=28 € \Pi C$

en .................. exemplaire, soit un total de .$€$

$\square$ Par chèque, à l'ordre de EDP Sciences

$\square$ Par carte bancaire :

$\square$ Visa $\square$ Eurocard/Mastercard

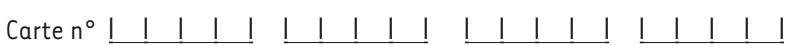

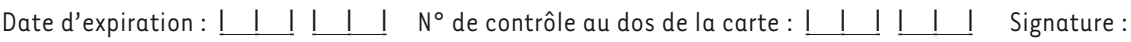

